\title{
Spin structure of the nucleon (theory)
}

\author{
Ulf-G. Meißner ${ }^{1,2, \star}$ and Akaki Rusetsky ${ }^{1, \star \star}$ \\ ${ }^{1}$ Universität Bonn, Helmholtz Institut für Strahlen- und Kernphysik, Bethe Center for Theoretical Physics, D-53115 Bonn, Germany \\ ${ }^{2}$ Forschungszentrum Jülich, Institute für Kernphysik (IKP-3), Institute for Advanced Simulation (IAS-4) and Jülich Center for \\ Hadron Physics, D-52145 Jülich, Germany
}

\begin{abstract}
We review the achievements of the project C.3, that dealt with the formulation and application of effective field theories including the $\Delta$ and Roper resonances as well as vector mesons. A particular focus was the calculation of finite volume effects and chiral extrapolations for lattice QCD.
\end{abstract}

\section{Introduction and motivation}

The project C.3 has been funded just for the first funding period of the SFB/TR 16. The PIs were Ulf Meißner and Akaki Rusetsky. The project was focused on the theory of the nucleon spin structure and expected to support the electroproduction experiments of the proposed project C.1. As this project was not funded, the focus $\mathbf{C . 3}$ shifted to the study of chiral extrapolations and finite volume effects, with a special emphasis on the inclusion of the $\Delta$ resonance and performing ground-breaking work of effective field theories (EFTs) with spin-1 fields and on the inclusion of the Roper resonance. The work on finite volume effects was continued in the second and third funding period in project A.6 "Baryon resonances in a finite volume" (PIs: Ulf Meißner and Akaki Rusetsky) whereas the work on unstable states in EFT was taken up again in the third funding period in the project C.9 "Chiral dynamics of/with unstable states" (PIs: Evgeny Epelbaum, Hermann Krebs and Ulf Meißner). Therefore, despite the re-orientation of the original project $\mathbf{C . 3}$, it has contributed significantly to the success of the SFB/TR 16.

Our contribution is organized as follows: Section 2 summarizes the results on including the $\Delta$-resonance in baryon chiral perturbation theory and making contact to lattice QCD through chiral extrapolations and finite volume methods. Sec. 3 is devoted to the studies of virtual Compton scattering off the nucleon, that were originally at the heart of this project and have later been taken up again in project C.9. The groundbreaking work on chiral extrapolations for the the low-lying baryons and the nucleon axial-vector coupling is discussed in Sec. 4. Sec. 5 deals with the developments of EFTs for the inclusion of vector mesons and the Roper resonance, that have been the trigger of many works by other groups in later years.

Note the important disclaimer: This is the review of the project $\mathbf{C . 3}$ but not a general review of the various

\footnotetext{
^e-mail: meissner@hiskp.uni-bonn.de

${ }^{\star}$ e-mail: hammer@theorie.ikp.physik.tu-darmstadt.de
}

fields to which this research contributed. Therefore, only the papers that resulted from this research are quoted, with the exception of a few external papers and of course, in case data were involved, with proper reference to the experimental papers. The links to the other projects are mentioned in passing, but no effort is made to discuss these in any detail.

\section{Effective field theory studies for spin-3/2 particles}

To discuss the spin structure of the nucleon in baryon chiral perturbation theory (CHPT), the inclusion of the $\Delta(1232)$ resonance as an effective degree of freedom is mandatory, see e.g. the detailed discussion in the review [1]. In Ref. [2], we have given an improved formulation of the covariant small scale expansion (SSE), i.e. an effective field theory (EFT) [3] with explicit spin$3 / 2$ (delta) degrees of freedom, counting the nucleon-delta mass splitting $m_{\Delta}-m_{N}$ as an additional small parameter, thus $\epsilon \in\left\{p / \Lambda, M_{\pi} / \Lambda,\left(m_{\Delta}-m_{N}\right) / \Lambda\right\}$ (with $\Lambda \approx 1 \mathrm{GeV}$ the scale of chiral symmetry breaking), and requiring consistency with the chiral limit of QCD (decoupling constraints). Within this approach, we have calculated the nucleon and the delta mass to fourth order $O\left(\epsilon^{4}\right)$ (complete one-loop calculation). We have analyzed lattice data from the MILC [4] and QCDSF [5] collaborations and demonstrated that the available lattice data combined with our knowledge of the physical values for the nucleon and delta masses lead to consistent chiral extrapolation functions for both observables up to fairly large pion masses, see Fig. 1. This holds in particular for very recent data on the delta mass from the QCDSF collaboration. The resulting pion-nucleon sigma term is $\sigma_{\pi N}=48.9 \mathrm{MeV}$. This first quantitative analysis of the quark-mass dependence of the structure of the $\Delta(1232)$ in full QCD within chiral effective field theory suggests that the nucleon-delta mass-splitting in the chiral limit, $\left(m_{\Delta}-m_{N}\right)_{0}=0.33 \mathrm{GeV}$, is slightly larger 


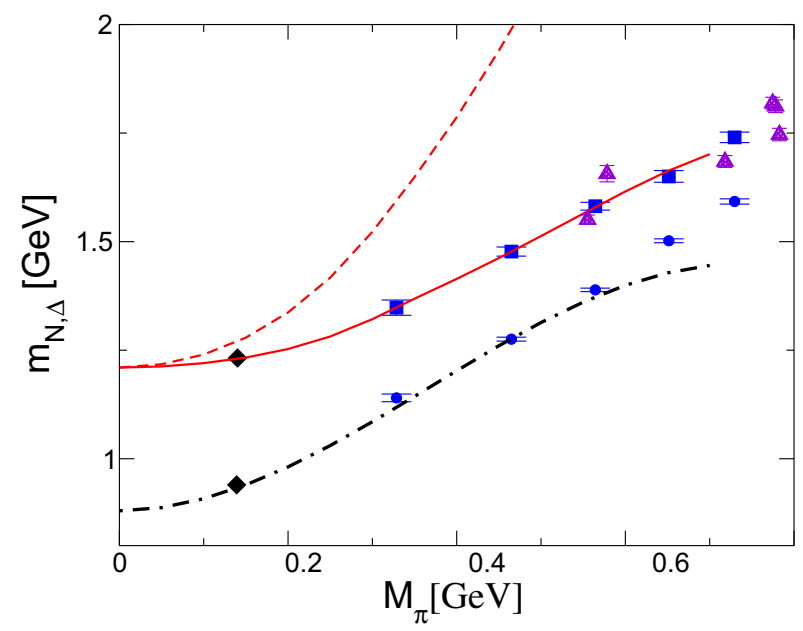

Figure 1. The nucleon mass (dot-dashed line) and the (real part of the) delta mass (solid line) as a function of the pion mass. The filled diamonds denote their physical values at the physical pion mass. The dashed line is the chiral extrapolation for the $\Delta$ based on SU(4). The filled squares and circles are the MILC data. The filled triangles are the recent data from QCDSF.

than at the physical point, $\left(m_{\Delta}-m_{N}\right)_{\text {phys }}=0.29 \mathrm{GeV}$. Further analysis of simultaneous fits to nucleon and delta lattice data are needed for a precision determination of the properties of the first excited state of the nucleon. Furthermore, the large deviation from SU(4) symmetry found in this work needs to be understood better and theoretical error bars have to be supplied, based on lattice results at lower quark masses. Clearly, these results should be considered indicative since the lattice data have been obtained at too high quark masses for a truely quantitative comparison with a small theoretical uncertainty.

As pointed out by Lüscher [6], the extraction of resonance properties from finite volume simulations is tricky. In particular, in a finite volume one only has discrete energy levels, and resonances can not be connected to any particular energy level measured on the lattice. Rather, in the vicinity of a resonance, the phenomenon of an avoided level crossing appears [7]. This allows one to extract the resonance mass and width (for well isolated resonances). This framework had before only be considered for the most simple cases like scalar field theories. To connect to the physical baryon spectrum, we have studied in Ref. [8] the extraction of Delta-resonance parameters from lattice data for small quark masses, corresponding to the case of an unstable Delta. To this end, we calculated the spectrum of the correlator of two Delta-fields in a finite Euclidian box up-to-and-including $O\left(\epsilon^{3}\right)$ in the small scale expansion using infrared regularization. On the basis of our numerical study, we argue that the extraction of the parameters of the Delta-resonance (in particular, of the mass and the pion-nucleon-delta coupling constant) from the measured volume dependence of the lowest energy levels should be feasible. For illustration, the dependence of the energy levels on the pion-nucleon-delta coupling is displayed in Fig. 2. This work has laid the foundation for the

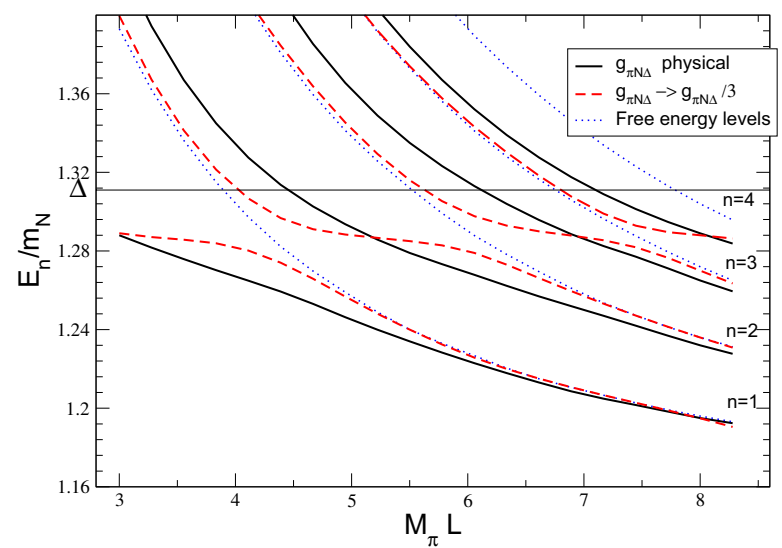

Figure 2. The dependence of the energy levels in a finite box on the box size $L$ for different values of the coupling constant $g_{\pi N \Delta}$. The avoided level crossing, which is clearly seen at small values of $g_{\pi N \Delta}$ (dashed lines), is washed out for the physical value of this coupling constant (solid lines). For comparison, we also display the free energy levels (dotted lines). It is seen that the energy levels in the presence of the interaction interpolate between different free energy levels. As expected, an abrupt change emerges in the vicinity of the resonance energy (the resonance position corresponds to the solid horizontal line).

new project A.6 (see that project for a more detailed discussion of these issues).

\section{Virtual Compton scattering off the nucleon}

In earlier work with Bernard and Hemmert [9], we had calculated the spin structure functions of the nucleon, in particular their lower moments, as measured in double virtual Compton scattering off nucleons based on covariant nucleon CHPT (using infrared regularization) to fourth order in the chiral expansion, supplemented by tree level delta and vector meson contributions. At that order, no undetermined parameters appear and the predictions are scrutinized by the data from Jefferson Laboratory for the proton and neutron spin structure function $g_{1}$ down to photon virtualities as low as $Q^{2} \simeq 0.05 \mathrm{GeV}^{2}$ (experiments with even lower values of $Q^{2}$ having been performed and are presently being analyzed). We have extended these calculations based on our covariant version of the SSE. This work was done in collaboration with M. Dorati from Pavia and T. Hemmert from Munich, and was later published by the Munich group in Ref. [10]. In that paper, the first moments of the parity-even Generalized Parton Distributions (GPDs) in a nucleon, corresponding to six (generalized) vector form factors, were calculated. The analysis was performed at leading-one-loop order in baryon CHPT, predicting both the momentum and the quark-mass dependence for the three (generalized) isovector and (generalized) isoscalar form factors, which are currently under investigation in lattice QCD analyses of baryon structure. We also studied the limit of vanishing four-momentum transfer where the GPD-moments reduce to the well known moments of Parton Distribution 


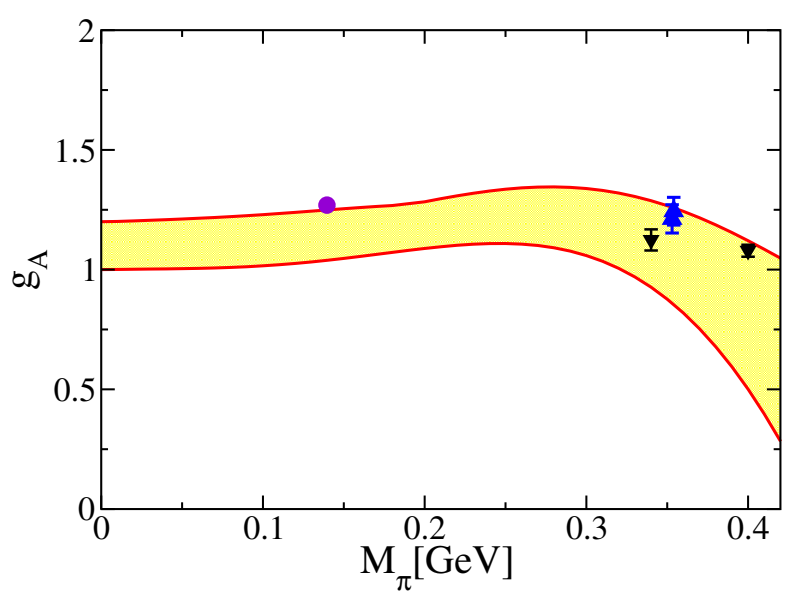

Figure 3. Pion mass dependence of the axial-vector coupling lattice results from LHPC/MILC (triangles) [12] and QCDSF (inverted triangles) [5] in comparison to the two-loop CHPT result (filled area). The circle is the physical point.

Functions (PDFs). For the isovector moment $\langle x\rangle_{u-d}$ this baryon CHPT calculation predicted a new mechanism for chiral curvature, connecting the high values for this moment typically found in lattice QCD studies for large quark masses with the smaller value known from phenomenology. Likewise, we analyzed the quark-mass dependence of the isoscalar moments in the forward limit and extracted the contribution of quarks to the total spin of the nucleon.

\section{Chiral extrapolations}

Related to the work on the delta and the nucleon mass, we have also considered the chiral expansion of various other observables. In Ref. [11] we have analyzed the nucleon axial-vector coupling $g_{A}$ to two loops in chiral perturbation theory making use of renormalisation group methods. This was motivated by the fact that while the one-loop representation of $g_{A}$ shows a strong pion mass dependence, the lattice results of various collaborations (MILC/LHPC, QCDSF, RBC, ...) are essentially independent of the pion mass for a large range of (unphysical) values. We show that chiral extrapolations based on this representation require lattice data with pion masses below $300 \mathrm{MeV}$ for a small theoretical uncertainty, see Fig. 3 (the band is obtained by varying the low-energy constants at dimension four and five within the bounds given by naturalness and assuming that $g_{A}\left(M_{\pi}\right)$ stays flat as indicated by all lattice data). This clearly is a benchmark calculation of interest for upcomimg lattice data in the true chiral regime.

In Ref. [13], we have calculated the baryon octet masses to fourth order in CHPT employing dimensional and cut-off regularization. We analyze the pion and kaon mass dependences of the baryon masses based on the MILC data from 2001 [14] and 2004 [15]. We show that chiral perturbation theory gives stable chiral extrapolation functions for pion (kaon) masses below 450 (500) MeV. The pion mass dependence of the nucleon mass is shown in Fig. 4. The pion-nucleon sigma term in $\mathrm{SU}(3)$ is also investigated, we find $\sigma_{\pi N}(0)=50.7-53.7 \mathrm{MeV}$. We note that some of the MILC 2004 data for the nucleon can not be described when one insists on the physical point (see Fig. 4) and that the pion mass dependence of the cascade mass of the MILC data is more pronounced than given by our extrapolation formula (having fixed the parameters on the nucleon).

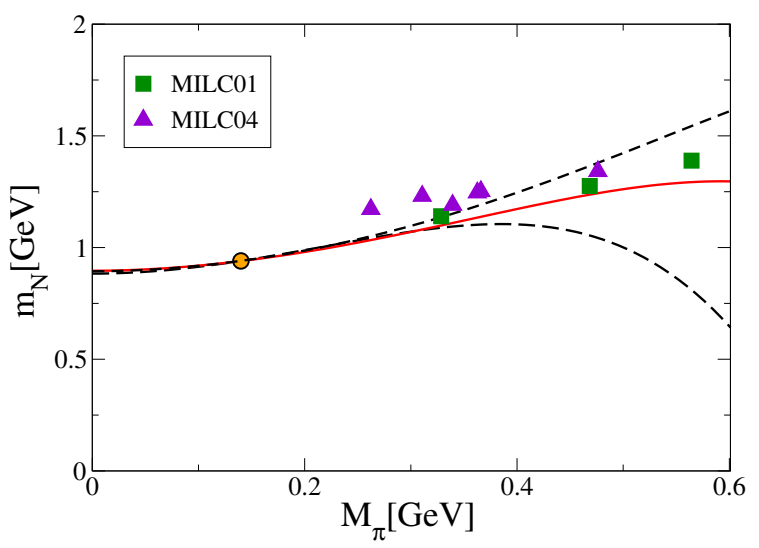

Figure 4. Pion mass dependence of the nucleon mass in SU(3). The red solid line gives the best fit as discussed in the text and the black dashed lines give the theoretical uncertainty under the constraint that the nucleon mass takes its physical value for the physical quark masses. Green squares: MILC 2001 data [14], purple triangles: MILC 2004 data [15]. The circle stands for the physical values.

\section{Effective field theories for excited states}

The experience obtained in dealing with the $N \Delta$-splitting in the SSE allowed us to consider also the pertinent EFTs for other excited hadrons. In Ref. [16], we have extended the method of infrared regularization to spin- 1 fields. This is an important step in the formulation of an EFT for such instable particles with a consistent power counting. As applications of this scheme, we have worked the chiral extrapolation of the rho meson mass, which takes the form

$$
M_{\rho}=M_{\rho}^{0}+c_{1} M_{\pi}^{2}+c_{2} M_{\pi}^{3}+c_{3} M_{\pi}^{4} \ln \left(\frac{M_{\pi}^{2}}{M_{\rho}^{2}}\right)+O\left(M_{\pi}^{4}\right),
$$

with $M_{\rho}^{0}$ the rho-meson mass in the chiral limit and the $c_{i}$ are combinations of low-energy constants of the effective chiral Lagrangian of Goldstone bosons and vector mesons. We have performed various fits of the existing lattice data from the CP-PACS collaboration, see Fig. 5. The chiral extrapolation function Eq. (1) has been used by many lattice groups after it was proposed in our work. We have also analyzed the pion-rho sigma term.

In the paper [18], we have presented an extension of the infrared regularization scheme that allows for an inclusion of explicit (vector and axial-vector) meson resonances in the single-nucleon sector of CHPT. For the processes we have considered here, the meson resonances do not appear as external particles, and the corresponding power counting rules for the internal resonance lines is set up such that the resonance four-momentum is considered 


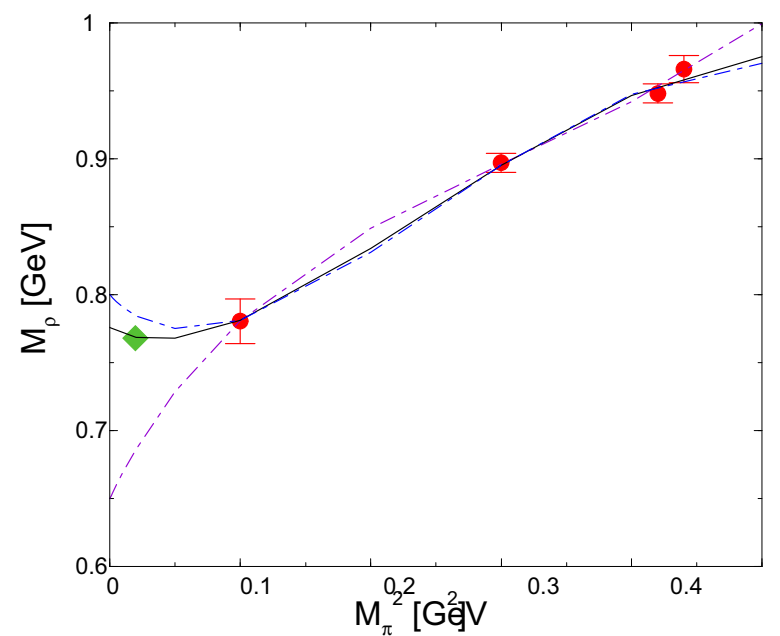

Figure 5. The rho meson mass $M_{\rho}$ as a function of the light quark mass, $M_{\pi}^{2} \sim\left(m_{u}+m_{d}\right)$. The solid (dot-dashed) line(s) refers to fit $1(2,3)$ as described in the text. The lattice data are from CPPACS [17]. The diamond denotes the physical rho mass.

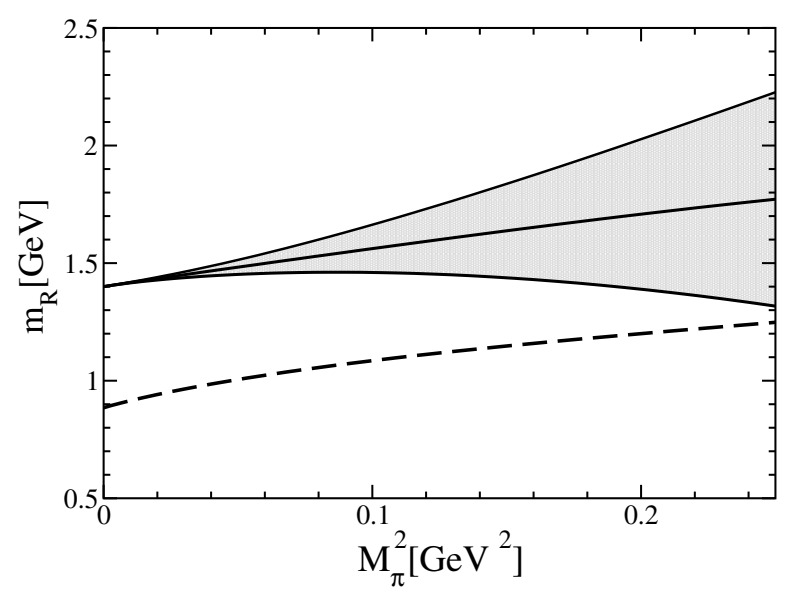

Figure 6. Quark mass dependence of the Roper mass for different parameter sets of the dimension two and four LECs within natural bounds (hatched area bounded by the solid curves). The lower dashed curve represents the quark mass dependence of the nucleon for standard values of the corresponding LECs: $c_{1}=-0.9, c_{2}=3.2, c_{3}=-3.45, e_{1}=-1.0$ (in appropriate units of $1 / \mathrm{GeV}$ ). The theoretical uncertainty for the nucleon mass is not shown in this plot (it is similar to the one for the Roper).

to be small compared to its mass. The infrared regularization scheme extracts the part of the one-loop graphs to which this power counting scheme applies (for any value of the dimension parameter $d$ used in dimensional regularization), while the remaining parts of the loop graphs will in general violate the power counting requirements, but can be absorbed in a renormalization of the local terms of the effective Lagrangian.

In Ref. [19], we have analyzed the quark mass dependence of the Roper mass (the real part of the self-energy) to one-loop order in covariant baryon chiral perturbation theory. The pertinent effective Lagrangian is spelled out in the Appendix. The loop integrals are evaluated using an extension of infrared regularization for two well separated large mass scales (as it is roughly fulfilled in nature since $m_{N}^{2} / m_{R}^{2} \simeq 1 / 2.4$ ). This method preserves chiral symmetry and establishes a chiral counting scheme. The derived chiral expansion of the Roper mass may prove useful for chiral extrapolations of lattice data. For couplings of natural size the quark mass dependence of the Roper mass is similar to the one of the nucleon, see Fig. 6. In particular, the derived quark mass dependence does not show a strong variation for small masses as indicated by some lattice results. We are presently extending these studies to a threescale problem to also analyse the decay $S_{11}(1535) \rightarrow N \eta$ and the relative quark mass dependence of the Roper and the $S_{11}$ mass.

We would like to thank our collaborators Véronique Bernard, Bugra Borasoy, Peter Bruns, Matthias Frink, Thomas Hemmert, Randy Lewis and Ilka Scheller. The work reported here would not have been possible without the support from the Deutsche Forschungsgemeinschaft within the SFB/TR16.

\section{A Effective Lagrangian of the nucleon-Roper-pion system}

Since the Roper is the first even-parity excited state of the nucleon, the construction of the chiral SU(2) effective Lagrangian follows standard procedures, see e.g. [20]. The effective Lagrangian relevant for our calculation is

$$
\mathcal{L}=\mathcal{L}_{0}+\mathcal{L}_{R}+\mathcal{L}_{N R},
$$

with the free part

$$
\mathcal{L}_{0}=i \bar{N} \gamma_{\mu} D^{\mu} N-M_{N} \bar{N} N+i \bar{R} \gamma_{\mu} D^{\mu} R-M_{R} \bar{R} R,
$$

where $N, R$ are nucleon and Roper fields, respectively, and $M_{N}, M_{R}$ the corresponding baryon masses in the chiral limit. $D_{\mu}$ is the chiral covariant derivative, for our purpose we can set $D_{\mu}=\partial_{\mu}$, see e.g. [20] for definitions. The pion-Roper coupling is given to leading chiral order by

$$
\mathcal{L}_{R}^{(1)}=\frac{1}{2} g_{R} \bar{R} \gamma_{\mu} \gamma_{5} u^{\mu} R,
$$

with an unknown coupling $g_{R}$ and the superscript denotes the chiral order. We have use $g_{R}=1.0$ as explained in Ref. [19]. The pion fields are collected in $u_{\mu}=-\partial_{\mu} \pi / f_{\pi}+$ $O\left(\pi^{3}\right)$, where $f_{\pi}$ is the pion decay constant in the chiral limit. At next-to-leading order, the relevant terms in $\mathcal{L}_{R}$ are (we work in the isospin limit $m_{u}=m_{d}$ and neglect electromagnetism)

$$
\begin{aligned}
\mathcal{L}_{R}^{(2)} & =c_{1}^{*}\left\langle\chi_{+}\right\rangle \bar{R} R-\frac{c_{2}^{*}}{8 M_{R}^{2}} \bar{R}\left(\left\langle u_{\mu} u_{\nu}\right\rangle\left\{D^{\mu}, D^{\nu}\right\}+\text { h.c. }\right) R \\
& +\frac{c_{3}^{*}}{2}\left\langle u_{\mu} u^{\mu}\right\rangle \bar{R} R,
\end{aligned}
$$

where $\chi_{+}$is proportional to the pion mass and induces explicit chiral symmetry breaking. Further, \langle\rangle denotes the trace in flavor space. For a complete one loop calculation we also need the fourth order effective Lagrangian, more precisely, the term

$$
\mathcal{L}_{R}^{(4)}=-\frac{e_{1}^{*}}{16}\left\langle\chi_{+}\right\rangle^{2} \bar{R} R .
$$


The interaction piece between nucleons and the Roper reads

$$
\mathcal{L}_{N R}^{(1)}=\frac{1}{2} g_{N R} \bar{R} \gamma_{\mu} \gamma_{5} u^{\mu} N+\text { h.c. . }
$$

The coupling $g_{N R}$ can be determined from the strong decays of the resonance $R$, its value is $g_{N R}=0.3 \ldots 0.4$. In principle a term of the form

$$
i \lambda_{1} \bar{R} \gamma_{\mu} D^{\mu} N-\lambda_{2} \bar{R} N+\text { h.c. }
$$

is possible, but applying the equations of motion removes the first term (and its hermitian conjugate) such that we are left with the terms $\bar{R} N$ and $\bar{N} R$. These terms induce mixing between the nucleon and Roper fields, but diagonalization of the $N-R$ mass matrix does not lead to new operator structures and its effect can be completely absorbed into the couplings already present in the Lagrangian. We can thus safely work with the Lagrangian in Eq. (2). A complete one-loop calculation involves tree graphs with insertion of chiral dimension two and four and one-loop graphs with at most one insertion from $\mathcal{L}_{R}^{(2)}$.

\section{References}

[1] V. Bernard, Prog. Part. Nucl. Phys. 60 (2008) 82 [arXiv:0706.0312 [hep-ph]].

[2] V. Bernard, T. R. Hemmert and U.-G. Meißner, Phys. Lett. B 622 (2005) 141 [arXiv:hep-lat/0503022].

[3] T. R. Hemmert, B. R. Holstein and J. Kambor, J. Phys. G 24 (1998) 1831 [hep-ph/9712496].

[4] C. W. Bernard et al., Phys. Rev. D 64, 054506 (2001) [arXiv:hep-lat/0104002].

[5] G. Schierholz, private communication; QCDSF collaboration, in preparation.
[6] M. Lüscher, Commun. Math. Phys. 105 (1986) 153.

[7] U.-J. Wiese, Nucl. Phys. Proc. Suppl. 9 (1989) 609.

[8] V. Bernard, U.-G. Meißner and A. Rusetsky, Nucl. Phys. B 788 (2008) 1 [hep-lat/0702012 [HEP-LAT]].

[9] V. Bernard, T. R. Hemmert and U.-G. Meißner, Phys. Rev. D 67 (2003) 076008 [hep-ph/0212033].

[10] M. Dorati, T. A. Gail and T. R. Hemmert, Nucl. Phys. A 798 (2008) 96 [nucl-th/0703073].

[11] V. Bernard and U.-G. Meißner, Phys. Lett. B 639 (2006) 278 [arXiv:hep-lat/0605010].

[12] R. G. Edwards et al. [LHPC Collaboration], Phys. Rev. Lett. 96 (2006) 052001 [arXiv:hep-lat/0510062].

[13] M. Frink, U.-G. Meißner and I. Scheller, Eur. Phys. J. A 24 (2005) 395 [arXiv:hep-lat/0501024].

[14] C. W. Bernard et al., Phys. Rev. D 64 (2001) 054506 [arXiv:hep-lat/0104002].

[15] C. Aubin et al., Phys. Rev. D 70 (2004) 094505 [arXiv:hep-lat/0402030].

[16] P. C. Bruns and U.-G. Meißner, Eur. Phys. J. C 40 (2005) 97 [arXiv:hep-ph/0411223].

[17] S. Aoki et al. [CP-PACS Collaboration], Phys. Rev. D 60 (1999) 114508 [arXiv:hep-lat/9902018].

[18] P. C. Bruns and U.-G. Meißner, Eur. Phys. J. C 58 (2008) 407 [arXiv:0808.3174 [hep-ph]].

[19] B. Borasoy, P. C. Bruns, U.-G. Meißner and R. Lewis, Phys. Lett. B 641 (2006) 294 [arXiv:heplat/0608001].

[20] N. Fettes, U.-G. Meißner, M. Mojžiš and S. Steininger, Annals Phys. 283 (2000) 273 [Erratumibid. 288 (2001) 249] [arXiv:hep-ph/0001308]. 